\title{
THE THERMODYNAMIC DRIVING FORCE FOR RAFTING IN SUPERALLOYS
}

\author{
F. R. N. NABARRO ${ }^{1,2}$, C. M. CRESS ${ }^{1,2 \dagger}$ and P. KOTSCHY ${ }^{1}$ \\ ${ }^{1}$ Division of Materials Science and Technology, CSIR, PO Box 395, Pretoria 0001, and ${ }^{2}$ Condensed Matter \\ Physics Research Unit, University of the Witwatersrand, Private Bag 3, WITS 2050, Johannesburg, \\ South Africa
}

(Received 5 April 1995; in revised form 30 October 1995)

\begin{abstract}
Eshelby's energy-momentum tensor is used to provide an analytical expression for the driving force for rafting in the clastic rcgime in a superalloy with a high volume fraction of $\gamma^{\prime}$. The structure is modelled as a simple cubic array of $\gamma^{\prime}$ cubes separated by thin sheets of $\gamma$. During rafting, the $\gamma^{\prime}$ particles are constrained to remain tetragonal prisms. For tension along a cube axis, the driving force is proportional to the product of the tension $\sigma$, the fractional difference $\delta$ of lattice parameters of $\gamma^{\prime}$ and $\gamma$ and the fractional difference $m$ of their elastic constants $c_{11}-c_{12}$. As in the calculation of Pineau for an isolated spheroid, needles are formed when this product $\sigma \dot{\delta} m$ is positive. Two- and three-dimensional systems behave similarly. The initial plastic strain in $\gamma$ is anelastic and in principle reversible. When the plastic strain exceeds $m \delta$, platelets perpendicular to the stress axis are formed if the product $\sigma \delta$ is negative. Copyright C 1996 Acta Metallurgica Inc.
\end{abstract}

Résumé On utilise le tenseur énergie-impulsion d'Eshelby pour obtenir une expression analytique de la force motrice du phénomène de formation de radeaux (rafting) dans le régime élastique d'un superalliage à grand contenu volumétrique de $\gamma^{\prime}$. La structure se modèle en ordre simple cubique de cubes de $\gamma^{\prime}$ séparés par de minces lamelles de $\gamma$. Les particules de $\gamma^{\prime}$ sont contraintes à rester des prismes tetragonaux pendant le processus de déformation de radeaux. Sous tension au long d'un axe cubique, la force motrice est proportionelle au produit de la tension $\sigma$, la difference fractionnaire $\delta$ des paramètres de réseau de $\gamma^{\prime}$ et $y$ et la différence fractionnaire $m$ de leur constants élastiques $c_{11}-c_{12}$. Comme dans les calculs de Pineau pour un sphéroïde isolé, il se forme des aiguilles quand ce produit $\sigma \delta m$ est positif. Les systemes à deux dimensions et à trois dimensions se comportent pareillement. La déformation plastique initiale en $\gamma$ est anélastique et en principe réversible. Quand la déformation plastique surpasse $m \delta$, des plaques perpendiculaires à l'axe de l'effort se forment quand le produit $\sigma \delta$ est negatif.

Zusammenfassung-Der Energie-Impuls Tensor von Eshelby wird für die Darstellung eines anlytischen Ausdrucks der treibenden Kraft für die Floss-Strukturbildung im elastischen Bereich in einer Superlegierung mit hohem Volumenantcil von $\gamma^{\prime}$ verwendet. Dic Struktur ist also eine einfache kubische Anordnung von $\gamma^{\prime}$-Würfeln, welche durch dünne Schichten von $\gamma$-Phase voneinander getrennt sind, modelliert. Während der Floss-Strukturbildung müssen die $\gamma^{\prime}$ Teilchen tetragonale Prismen bleiben. Für die Spannung entlang der kubischen Achse ist die treibende Kraft proportional zum Produkt der Spannung $\sigma$, dem relativen Untershied $\delta$ der Gitterparameter von $\gamma^{\prime}$ and $\gamma^{\prime}$, und der fraktionalen Differenz $m$ ihrer elastischen Konstanten $c_{11}-c_{12}$. Wie in der Berechnung von Pineau für einen isolierten Spherö̈d, formen sich Nadeln falls dieses Produk positiv ist. Zwei- und dreidimensionale Systeme verhalten sich ähnlich. Die anfängliche plastische Dehnung in $\gamma$ ist anelastisch und in Prinzip umkehrbar. Falls die plastische Dehnung $m \delta$ überschreitet und das produkt $\sigma \delta$ negativ ist, formen sich Plättchen senkrecht zur Spannungsachse.

\section{INTRODUCTION}

To a very good approximation, a modern superalloy is composed of cubical particles of an alloy having the ordered $\mathrm{Ll}_{2}$ structure, conventionally labelled $\gamma^{\prime}$, which lic with lattice coherence in a matrix of an alloy of similar composition having the disordered face-centred cubic structure, conventionally labelled $\gamma$. The particles, again to a very good approximation, form a simple cubic array with its axes parallel to the axes of the underlying cubic crystal structures.

$\nmid$ Present address: Department of Physics and Astronomy, Columbia University, New York, NY, U.S.A.
If a typical alloy is stressed by tension along the [001] axis at a high temperature, it undergoes plastic deformation by the motion of dislocations. This process of creep causes the sample to elongate along [001] and to contract along [001] and [010]. At the same time, a process called rafting may be observed [1-3]. Each cubical particle shortens along [001] and expands along [100] and [010]. When the deformation of the particles reaches about $15 \%$, particles adjacent along [100] or [010] may meet and weld together, producing an extended raft-shaped particle of $\gamma^{\prime}$. If the stress applied along [001] is a compression rather than a tension, the individual particles deform so that 
their short axes lie along [100] or [010], or they may form needles directed along [001] [3, 4]. In other alloys, the effects of tension and compression are interchanged [5].

The processes of rafting and of creep by the motion of dislocations are essentially distinct. In the lower part of the usual range of testing temperatures, considerable dislocation motion and multiplication occurs without any observable rafting [e.g. 6, 7]. On the other hand, at high temperatures and under low stresses, the distortional strain of each particle during rafting is of the order of $15 \%$ while the total crecp strain of the sample as a whole is only about $0.02 \%$. Moreover, the deformation of each particle under typical conditions of rafting has the opposite sense to the bulk deformation of the sample [8].

We believe that rafting is essentially a diffusional process in which lattice coherence is maintained. The boundaries of each ordered $\gamma^{\prime}$ region move, but the volume of the region remains constant. We therefore consider the driving force for rafting in the absence of plastic deformation mediated by dislocations. It is, of course, possible that the rate of rafting may be increased if dislocations are present to provide mechanisms of easy diffusion and it is certain that the stress concentration produced by plastic flow in the $\gamma$ matrix can influence both the rate and the direction of rafting.

The case of an isolated particle whose shape can be adequately represented by a spheroid was solved exactly within the approximation of isotropic elasticity by Pineau [9], using the methods developed by Eshelby $[10,11]$. Even though Pineau simplified the calculations by assuming that the matrix and the inclusion have the same Poisson ratios, the formulae are complicated, and most of the results are displayed only numerically.

Fundamental parameters are the elastic moduli of the matrix and of the inclusion, $E_{\mathrm{m}}$ and $E_{\mathrm{p}}$ respectively. We shall write

$$
E_{\mathrm{p}} / E_{\mathrm{m}}=1+m,
$$

and assume that $m$ is small, so that terms in $m^{2}$ may be neglected. In practice, $m$ is of the order of 0.15 .

Further fundamental parameters are the unstrained lattice parameters $a_{\mathrm{m}}$ and $a_{\mathrm{p}}$. We define the misfit parameter $\delta$ by

$$
\delta=2\left(a_{\mathrm{p}}-a_{\mathrm{m}}\right) /\left(a_{\mathrm{p}}+a_{\mathrm{m}}\right) .
$$

The applied stress $\sigma$ causes an elastic elongation $\sigma / E_{\mathrm{m}}$, and its importance may be measured by the ratio of this elongation to the misfit $\delta$. We write

$$
\Sigma=\sigma / E_{\mathrm{m}} \delta \text {. }
$$

Pineau's calculations neglect the interfacial energy of the two phases, which means that they apply to "large" particles. The dimensions of a particle for which surface energy is of comparable importance to elastic energy are of the same order as the radius of curvature of the rounded edges of a "large" particle. This rounding is not pronounced in micrographs of the $\gamma^{\prime}$ particles in most superalluys, so that these particles can reasonably be treated as "large".

The strength of Pineau's calculation lies in the fact that Eshelby's formulae allow him to treat values of $m$ ranging from -1 to $\infty$, and values of $\Sigma$ ranging from $-\infty$ to $\infty$. One weakness is that the numerical calculations become imprecise when these parameters are small, which is usually the case of practical interest. Another weakness is the use of isotropic clasticity, because the $\gamma$ and $\gamma^{\prime}$ phases actually involved show extreme elastic anisotropy, with $2 c_{44} /\left(c_{11}-c_{12}\right)$ of the order of 3.5 . Finally, with a typical $\gamma^{\prime}$ volume fraction of over $60 \%$, the cubes of $\gamma^{\prime}$ are separated by sheets of $\gamma$ whose thickness is only about 0.15 of the side of each cube; the approximation that the $\gamma^{\prime}$ cubes are isolated is no longer plausible.

The Fourier transform method of Khachaturyan et al. [12] allows some problems to be solved exactly for a cubical array in anisotropic elasticity. However, their treatment is limited to the case in which matrix and precipitates have the same elastic constants. Our own analysis confirms the results of Pineau for an isolated precipitate particle: there is no driving force for rafting under external stress in the elastic regime if matrix and precipitate have the same elastic constants. Similarly, our prediction that, for small values of $\Sigma$ and $m$, rafts are formed normal to the stress axis if $m \Sigma$ is negative, and needles along the stress axis if $m \Sigma$ is positive, agrees with Pineau's results.

By numerical analysis, Socrate and Parks [13] calculated the driving force for rafting in a regular cubical array both with and without allowance for plastic flow in the $\gamma$ matrix. The calculations are carried out in a generalized plane strain two-dimensional model, allowing a uniform displacement in the third dimension. It does not seem obvious that a two-dimensional model will successfully predict the behaviour in the real three-dimensional case. In the absence of plastic flow, their results in the elastic regime agree with those of Pineau for the isolated three-dimensional inclusion: the nature of rafting is determined by the sign of the product $m \Sigma$. These predictions do not always agree with experiment. After plastic strain in the $\gamma$ matrix of the order of $2 \frac{1}{2}$ times the initial misfit, the predicted directions of rafting agree with those observed. Under these conditions, the initial misfit and the elastic properties cease to be important. If the stress is tensile, $\gamma$ material is flowing from the transverse sheets to the normal sheets. This implies that there is a large hydrostatic tension in the middle of the normal sheets of $\gamma$. If $\delta$ is positive, this tension will be relieved if the $\gamma^{\prime}$ cube elongates along the tensile axis. Thus the condition for the formation of rafts normal to the tensile axis is that $\sigma \delta$, or equivalently $\Sigma$, is negative. This prediction is confirmed by the 
calculations of Socrate and Parks, and is in agreement with experinnent.

\section{SOME GENERAL RESULTS}

We now discuss some general results in linear elasticity which may either assist the analysis or provide criteria which the results of the analysis must satisfy. Most of them concern homogeneous elastic bodies in which a region undergoes a uniform expansion, not necessarily isotropic, while remaining coherent with the matrix. If the elastic constants are unaltered by the transformation, the transformed region is called an inclusion; if the elastic constants are altered, the region is called an inhomogeneity. Some results apply in the presence of external stresses, others to internal stresses alone.

\subsection{Colonnetti's theorem}

The most general result is Colonnetti's theorem [14]. If an arbitrary body is subjected to both internal and external stresses, the internal energy contains no cross terms between the internal and the external stresses. The proof is intuitive. Take the body internally stressed. Its surface is free from tractions. Impose surface displacements which increase slowly from zero to their final values. By the principle of superposition, the surface tractions increase in linear proportion to these imposed displacements. Thus the work done by the surface tractions is proportional to the square of the surface displacement, or to the square of the tractions, and this work is stored in the body. The internal energy is the sum of the initial internal energy and of this work.

\subsection{Consequences of cubic symmetry}

We consider an infinite medium of cubic symmetry, free from external stresses, in which there is an elastic inhomogeneity having the form of a parallelepiped or a spheroid whose principal semi-axes $a, b$ and $c$ are directed along cubic axes. Then, for a given volume of the inhomogeneity, the elastic energy is stationary when $a=b=c$. (In the two-dimensional case the result follows at once from symmetry, because the region of dimensions $a \times b$ is converted into a region of dimensions $b \times a$ by a rotation of $\pi / 2$, which is a symmetry operation of the system.)

Let the elastic energy of the system be $U(a, b, c)$ Then, when $a=b=c$,

$$
\frac{\partial U}{\partial a}=\frac{\partial U}{\partial b}=\frac{\partial U}{\partial c}=\operatorname{grad}_{0} U
$$

and so, to first order,

$$
\delta U=\operatorname{grad}_{0} U(\delta a+\delta b+\delta c),
$$

but, at constant volume, to first order,

$$
\delta a+\delta b+\delta c=0,
$$

and the result follows. In the absence of external stress, a spherical or cubical inhomogeneity is always in equilibrium, stable or unstable.

\subsection{Transformation without change of elastic con- stants}

The next two results concern a finite homogeneous body in which a region, not necessarily small in comparison with the whole body, undergoes a homogeneous expansion, not necessarily isotropic, while retaining its elastic constants and its coherence with the matrix. The proofs will be published elsewhere [15].

2.3.1. The change of volume. Under the conditions stated, the volume of the body after the transformation has occurred is equal to the sum of the volumes of the unconstrained matrix and the unconstrained inclusion after transformation.

2.3.2. Work of external stress. If the transformation occurs under a uniform external stress, the work done by this stress during the transformation is equal to the work which would be done by this stress during the transformation of the otherwise unconstrained inclusion. It follows that a uniform external stress cannot influence the equilibrium shape of an inclusion which has the same elastic constants as the matrix.

Consider the process of rafting under an applied tensile stress $\sigma$. Initially, a symmetrical supercell (see Section 4) under zero stress has energy $U_{\mathrm{v}}$. When the stress is applied, the energy increases to $U_{\mathrm{o}}+g \sigma^{2}$, where $2 g$ is the total volume divided by the appropriate elastic constant. Rafting measured by $c$ is then initiated by transforming layers of $\gamma^{\prime}$ normal to $\sigma$ into $\gamma$. By Theorem 2.3.2, the work done by the external stress is the same as that which would be done if the $\gamma^{\prime}$ inclusion was isolated from $\gamma$. Rafting is then completed by transforming an equal volume of $\gamma$ in sheets parallel to $\sigma$ into $\gamma^{\prime}$. Theorem 2.3.2 again applies, and the work done in this step exactly cancels that done in the previous step. By Colonnetti's theorem (2.1) the total internal energy is the sum of the elastic energies of the internal and the external stress systems considered separately. By Theorem 2.2 the former is $U_{\circ}+O\left[\epsilon^{2}\right]$. Since the body is elastically homogeneous, the latter is still $g \sigma^{2}$. The "driving force" for rafting is measured by the difference between the total work done by the external stresses, which has been shown to be zero, and the increase of internal energy, which is $O\left[c^{2}\right]$. There is thus no driving force for rafting of the equiaxed configuration if $\gamma$ and $\gamma^{\prime}$ have the same elastic constants.

\subsection{Khachaturyan's theorem}

An important result is due to Khachaturyan [16]: an inclusion of given volume in an elastically anisotropic medium has minimum elastic energy if it is "rolled out" to give an infinite platelet of infinitesimal thickness whose habit is determined by the elastic anisotropy. 


\subsection{Transformation without change of rigidity}

For a homogeneous coherent region undergoing an isotropic expansion with no change in rigidity in an elastically isotropic matrix, the elastic energy (with or without external stress) depends only on the volume of the inclusion and not on its shape. This result is due to Goodier [17], and was rediscovered independently by Crum [18] and Robinson [19].

While a formal analytical proof is available, a qualitative argument allows an interesting extension: if the matrix is not infinite, but all parts of its free boundary are far from the inclusion in comparison with the dimensions of the dilated region, the change in volume is independent of the shape of the dilated volume. We may regard the dilated volume as composed of infinitesimal centres of dilatation. Within each centre, the centre itself has a purely dilatational field. Outside the centre the field is purely shear. Because the transformation occurs without change of shear modulus, the elastic field of each centre propagates uniformly through the matrix and through other centres. The centres may be displaced with respect to each other without any elastic interaction.

\section{A QUALITATIVE MEASURE OF THE DRIVING FORCE}

It is sometimes suggested that one can predict the sense of rafting in the elastic regime by the following simple argument: suppose, for example, that $\gamma^{\prime}$ has a larger lattice parameter than $\gamma$, that $\gamma^{\prime}$ is stiffer than $\gamma$, and that tension is applied along a cube axis. Then, on the lateral interfaces, the weaker $\gamma$ will be stretched by the applied stress so that its lattice parameter tits that of $\gamma^{\prime}$ better, while, neglecting Poisson contractions, the misfit on the transverse interfaces will not be affected. The misfit energy will be reduced, and all the more so if the lateral faces increase in area, i.e. needles are formed. The answer is right, but the argument is fallacious, because (1) Colonnetti's theorem shows that there are no cross terms in the total internal energy between the internal and the external forces, and it is just such a cross term which is considered here; and (2) rafting is driven by the difference between the work done by the loading machine and the increase in internal energy, and the present argument neglects the work done. The argument following equation (32) seems free from such objections.

\section{THF, MODE,}

We shall use a model in which identical cubical supercells (Fig. 1), each consisting of a centred $\gamma^{\prime}$ cube surrounded by a shell of $\gamma$, are stacked in an array which initially is simple cubic.

If a single supercell is dissected out, the misfit between $\gamma$ and $\gamma^{\prime}$ will, in general, cause the supercell to become barrel- or pincushion-shaped, even if $\gamma$ and

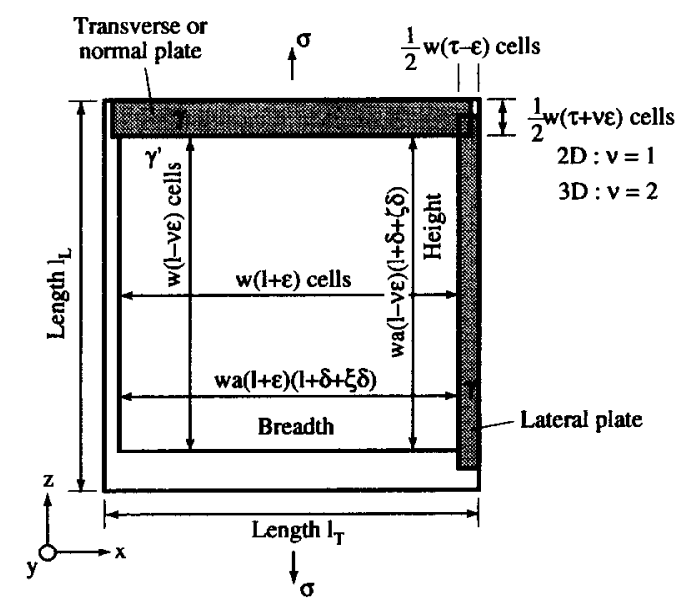

Fig. 1. Rafted unit supercell in a superalloy. The coherent interfaces have wa(1+c) or wa(1-vc) lattice cells; the unstrained lattice parameter of the matrix lattice is $a$.

$\gamma^{\prime}$ have the same elastic constants. Before the disassembled cells can be reassembled, they must be constrained by surface forces to have plane faces. These forces vary across each interface between supercells. They are not easy to calculate, and their stress fields, which we will call pincushion stresses, extend into the supercell for a distance which will not allow them to be neglected on the nearest $\gamma-\gamma^{\prime}$ interfaces. On the other hand, the specimen as a whole is initially free from external stress, and so the total force acting across any face of a supercell is initially zero. We shall argue that the presence of the pincushion stresses in the assembled system makes a negligible contribution to the thermodynamic driving force for rafting.

Let $\tau$ be the ratio of the thickness of a $\gamma$ sheet to the side of a $\gamma^{\prime}$ cube, and let $\delta$ be the lattice misfit between $\gamma$ and $\gamma^{\prime}$. Then, when $\tau$ is small, as we shall assume it to be, the strains involved in constraining this supercell to have planar faces are obviously proportional to $\delta \tau$. The work done in the process of constraint is then of the order of $\tau^{2}$, and can be neglected. We shall also neglect terms involving the product $m \tau$.

Since one question of interest is whether there are essential differences in behaviour between the twoand the three-dimensional cases, we treat the cases together, labelling the two-dimensional case by $v=1$ and the three-dimensional case by $v=2$. The longitudinal direction is that cube axis along which a tensile stress $\sigma$ may be applied, and there are $v$ transverse directions. We consider only the elastic regime in which coherence is maintained between $\gamma$ and $\gamma^{\prime}$ lattices, and take the unit supercell to have $2(v+1)$ sides each of $w(1+\tau)$ lattice cells. The $\gamma^{\prime}$ particle, originally a square or cube of $w^{v+1}$ lattice cells, has after rafting $w(1+\epsilon)$ cells in each of $v$ transverse directions, and, to maintain a constant 
volume fraction of $\gamma^{\prime}, w(1+\epsilon)^{-v} \approx w(1-v \epsilon)$ cells in the longitudinal direction.

Then (Fig. 1) the symmetrical unit supercell has two plates normal to the longitudinal direction which each are $\frac{1}{2} w(\tau+v \epsilon)$ cells thick and of transverse dimensions $w\left(1+\frac{1}{2} \epsilon+\frac{1}{2} \tau\right)$ cells. As shown in the figure, this last choice ensures that in the two-dimensional corners or three-dimensional edges the number of unoccupied cell sites is equal to the number of doubly-occupied sites. If the edges of the plates are bevelled, the sites in a supercell are each occupied exactly once. There are $2 v$ transverse plates each $\frac{1}{2} w(\tau-\epsilon)$ cells thick, of longitudinal length $w\left[1-\frac{1}{2} v \epsilon+\frac{1}{2} \tau\right]$ cells.

The side of the unit cell in $\gamma$ being $a$ and of that in $\gamma^{\prime}$ being $(1+\delta) a$, we assume that when the supercell is assembled in clastic equilibrium from a $\gamma^{\prime}$ block and $2(v+1) \gamma$ plates, the actual length of a transverse $\gamma-\gamma^{\prime}$ interface is $w a(1+\epsilon)(1+\delta+\xi \delta)$ while the actual length of a longitudinal interface is $w a(1-v \epsilon)(1+\delta+\zeta \delta)$.

If longitudinal strains are denoted by $e_{\mathrm{L}}$ and transverse strains by $e_{\mathrm{T}}$, the strains in the components are as follows:

in $\gamma$ :

$$
\begin{aligned}
& e_{\mathrm{iL}} / \delta=1+\zeta \\
& e_{\mathrm{r} T} / \delta=1+\xi
\end{aligned}
$$

in $\gamma^{\prime}$ :

$$
\begin{array}{r}
e_{y^{\prime} \mathrm{L}} / \delta=\zeta \\
e_{\gamma^{\prime} \mathrm{T}} / \delta=\xi
\end{array}
$$

\section{THE METHOD OF CALCULATION}

There are two possible approaches to the calculation. In the first, we calculate the internal energy as a function of the distortion $\epsilon$ to first order in $\epsilon$, calculate the work done by the external stress $\sigma$ during the distortion to order $\epsilon$, and take the difference, which gives the change in enthalpy. This procedure turns out to be analytically complicated. It is preferable to use the Eshelby energy-momentum tensor to calculate the thermodynamic force tending to move each interface, as was done by Socrate and Parks [13] in considering this problem.

Eshelby $[20,21]$ showed that in an elastically stressed body there is an effective (configurational) normal force $F$ on unit area of an interface, given by

$$
F=[W]-\mathbf{T} \cdot\left[\frac{\partial \mathbf{u}}{\partial n}\right]
$$

Here $[Y]$ represents the value of $Y$ on the side of the interface towards which the normal points minus its value on the other side, $W$ is the elastic energy density, $\mathbf{T}$ is the traction at the interface, and $\partial \mathbf{u} / \partial n$ is the normal gradient of the elastic displacement.
This formula, derived by the use of the elastic energy-montentum tensor, has an intuitive interpretation: when the interface sweeps out unit volume, the elastic energy changes by $\lceil W\rceil$, and the local stresses do work $\mathbf{T}[\partial \mathbf{u} / \partial n]$.

The formula simplifies in the present problem, because $\mathbf{T}$ is a purely normal stress $p_{\mathrm{n}}$ and $\partial \mathbf{u} / \partial n$ is a diagonal strain component $e_{\mathrm{n}}$. We may thus write

$$
F_{\mathrm{n}}=[W]-p_{\mathrm{n}}\left[e_{\mathrm{n}}\right]
$$

The unknown "pincushion" stresses which are required to constrain the supcrecll to have planar faces enter into both terms of equation (12), but we proceed to show that they make a negligible contribution to the final result. The terms in equation (12) are quadratic in the elastic field, and so the pincushion stresses enter both as their products with the pincushion strains and as their products with the misfit strains $\delta$ and with the strains of order $\sigma / c_{11}$ produced by the external stress. The pincushion stresses are of order $c_{11} \delta \tau$, and the corresponding strains are of order $\delta \tau$. The product of these two is of order $c_{11} \delta^{2} \tau^{2}$, and negligible. The contribution to the energy density $W$ of the product of the pincushion stresses and the strain produced by the external stress is of order $\sigma \delta \tau$, and the discontinuity $[W]$ of this contribution across an interface is of order $m \sigma \delta \tau$, and negligible. Similar arguments show that all but one of the cross terms in $p_{n}\left[e_{n}\right]$ may be neglected on each interface. The remaining term is the product of the contribution $c_{11} \delta \tau$ of the pincushion stresses to $p_{\text {n }}$ with the misfit strain $\delta$ normal to an interface. This product tends to move each interface outwards to produce a homogeneous unstrained cube of $\gamma^{\prime}$. However, in our model, rafting occurs at constant volume of $\gamma^{\prime}$, and the contributions of the transverse and the lateral interfaces to the total thermodynamic force for rafting cancel.

We approximate the stress distribution by assuming that the principal stress axes are everywhere parallel to the cube axes and that each principal component of stress is continuous across an interface which it meets normally. The values of these principal components are determined by requiring elastic equilibrium over the faces of each supercell and across the principal central sections of each supercell. While this approximate stress system ensures elastic equilibrium, it leads to discontinuities of strain in the corner and edge regions of each supercell. While the supplementary strains required to restore continuity are large of order $\delta$, they are confined to small parts of the supercell, and we assume that their effects are negligible.

Even with these approximations the problem is too complicated to be solved without the aid of computer algebra. This recourse to a computer has the disadvantages that one loses all physical insight, and that it is difficult to detect errors. We therefore adopted the following procedure: in a first approximation, Poisson contractions were neglected by 
putting $c_{12}=0$ in both $\gamma$ and $\gamma^{\prime}$. This is far from the practical situation, but is physically acceptable because the theoretical limits on Poisson's ratio are $\frac{1}{2}$ and -1 . This procedure completely decouples the longitudinal and transverse elastic fields, and leads to two sets of equations which are tractable manually. The analysis applics to either the two- or threedimensional case, and is outlined in Section 6. Then, in Section 7, the complete analysis for the three-dimensional case is outlined, and it is confirmed that setting $c_{12}=0$ in both media recovers the result of Section 6.

\section{POISSON CONTRACTION NEGLECTED}

In this illustrative calculation we neglect both the Poisson contractions and the pincushion stresses, but we consider both the two- and three-dimensional cases.

With these approximations, the strains in thickness are, in a transverse $\gamma$ plate

$$
e_{1 \mathrm{~T}} / \delta=0
$$

and in a normal $\gamma$ plate,

$$
e_{3 \mathrm{~L}}=\sigma / c_{11} \text {. }
$$

We now write down the condition that there should be no total force across a longitudinal central section of a supercell. We take

$$
c_{11}^{\prime}=(1+m) c_{11},
$$

and assume that $m$ and $\tau$ are of the same order of smallness, so that terms in $m^{2}$ and $m \tau$ can be neglected. We omit a factor $w^{v} a^{v} \mathcal{C}_{11}$. The condition is then, when $\epsilon=0$,

$$
v \tau(1+\xi)+(1+m)(1+\delta)^{v} \xi=0 .
$$

Since the strains involve $\xi$ in the form $\xi \delta$, and the strains are to be considered only to terms linear in $\delta$, the factor $(1+\delta)^{v}$ in equation (16) may be replaced by unity. Retaining only terms linear in $m$ and $\tau$, we find

$$
\xi=-v \tau \text {. }
$$

Similarly, the condition that the total force across a transverse central section of a supercell should be that produced by the applied stress is, for $\epsilon=0$,

$$
v \tau(1+\zeta)+(1+m)(1+\delta)^{r} \zeta=(1+\delta+\tau)^{v} \Sigma
$$

where

$$
\Sigma=\sigma / c_{11} \delta
$$

We note that $\Sigma$, unlike $m$ and $\tau$, is not assumed to be small.

According to equations (7) and (9), the strains contain terms in $\delta$ and $\delta \zeta$, and we wish to determine them to orders $\delta$ and $\sigma / c_{11}$, that is to say, to orders $\delta$ and $\delta \Sigma$. It is thus adequate to determine $\zeta$ to order zero in $\delta$, and $(1+\delta)$ in equation (18) may be replaced by unity. The solution, neglecting terms in $\epsilon^{2}$ and terms quadratic in $m$ and $\tau$, is

$$
\zeta=-v \tau+\Sigma-m \Sigma \text {. }
$$

Because of our assumption that $c_{12}=c_{12}^{\prime}=0$, the energy density depends only on the squares of the strains in cquations (10), (13) and (14). Using these equations together with equations (17) and (20) leads, on retaining only terms up to those quadratic in $\Sigma$ and only those linear in $m$ and $\tau$, to the results of equations (A1)-(A6) of the Appendix.

We insert these quantities in equation (12).

There are $2 v$ interfaces parallel to the stress axis. Each is of area $w^{v} a^{v}$, and moves a distance $\frac{1}{2} w a \epsilon$ in the direction of the outward normal to the $\gamma^{\prime}$ cube when the distortion $\epsilon$ occurs. The energy density outside the interface is

$$
W_{\mathrm{ol}}=\frac{1}{2} c_{11}\left[e_{\digamma \mathrm{L}}^{2}+(v-1) e_{\gamma \mathrm{T}}^{2}\right]
$$

where $e_{; \mathrm{L}}^{2}$ and $e_{; \mathrm{T}}^{2}$ are obtained from equations (Al) and (A2). This gives

$$
\begin{aligned}
W_{\mathrm{ol}}=\frac{1}{2} c_{11} \delta^{2}\left(v-2 v^{2} \tau\right. & +2 \Sigma-2 m \Sigma \\
& \left.-2 v \tau \Sigma+\Sigma^{2}-2 m \Sigma^{2}\right) .
\end{aligned}
$$

In calculating the energy density just inside the interface, we recognize that within the present approximation the interface is sufficiently close to the unstressed free surface of the supercell for there to be no normal stress or strain. The energy density is thus

$$
\boldsymbol{W}_{\mathrm{i} 1}^{r}=\frac{1}{2} c_{11}(1+m)\left[e_{\gamma^{\prime} \mathrm{L}}^{2}+(v-1) e_{\gamma \mathrm{T}}^{2}\right],
$$

where $e_{\% 1 .}^{2}$ and $e_{\% \mathrm{~T}}^{2}$ are obtained from equations (A3) and (A4), giving

$$
W_{\mathrm{il}}=\frac{1}{2} c_{11} \delta^{2}\left(-2 v \tau \Sigma+\Sigma^{2}-m \Sigma^{2}\right)
$$

On these interfaces $p_{n}=0$ and so

$$
\begin{aligned}
& {[W]-p_{\mathrm{n}}\left[e_{\mathrm{n}}\right]=} \\
& \quad \frac{1}{2} c_{11} \delta^{2}\left(v-2 v^{2} \tau+2 \Sigma-2 m \Sigma-m \Sigma^{2}\right) .
\end{aligned}
$$

This is the configurational pressure moving these interfaces outwards.

When rafting occurs, they sweep out a volume $v w^{v+1} a^{v+1} c$, and the energy released when they move is

$$
\frac{1}{2} v w^{v+1} a^{v+1} c_{11} \delta^{2} c\left[v-2 v^{2} \tau+2 \Sigma-2 m \Sigma-m \Sigma^{2}\right] .
$$

In the two sheets of $\gamma$ normal to the stress axis, the energy density is

$$
\begin{aligned}
W_{\mathrm{o} 3} & =\frac{1}{2} c_{11}\left(v e_{; \mathrm{T}}^{2}+e_{3 \mathrm{~L}}^{2}\right) \\
& =\frac{1}{2} c_{11} \delta^{2}\left(v-2 v^{2} \tau+\Sigma^{2}\right)
\end{aligned}
$$


while the energy density inside is

$$
\begin{aligned}
W_{\mathrm{i} 3} & =\frac{1}{2} c_{11}\left[(1+m) v e_{\gamma^{\prime} \mathrm{T}}^{2}+(1-m) \Sigma^{2}\right] \\
& =\frac{1}{2} c_{11}(1-m) \Sigma^{2} .
\end{aligned}
$$

On this interface,

$$
p_{\mathrm{n}}=c_{11} \delta \Sigma \text {. }
$$

The normal strain outside is $\delta \Sigma$, and that inside, allowing for the transformation strain, is $\delta+(1-m) \delta \Sigma$.

Thus on these interfaces

$$
\left[e_{\mathrm{n}}\right]=-\delta+m \delta \Sigma
$$

and

$$
[W]-p_{\mathrm{n}}\left[e_{\mathrm{n}}\right]=\frac{1}{2} c_{11} \delta^{2}\left(v+2 \Sigma-2 v^{2} \tau-m \Sigma^{2}\right) .
$$

These two sheets are also of area $w^{\prime \prime} a$, and during rafting they sweep out a volume $-v w^{v+1} a^{v+1} \epsilon$. The energy released when they move is

$$
-\frac{1}{2} v w^{v+1} a^{v+1} c_{11} \delta^{2} \epsilon\left[v-2 v^{2} \tau+2 \Sigma-m \Sigma^{2}\right] .
$$

The total energy release is the sum of equations (25) and (31), equal to

$\frac{1}{2} v w^{v+1} a^{v+1} c_{11} \delta^{2} \epsilon(-2 m \Sigma)=-v w^{v+1} a^{v+1} \delta \epsilon m \sigma$.

There is no essential difference between the cases of two dimensions $(v=1)$ and three dimensions $(v=2)$. Morenver, hoth agree with the terms linear in $\sigma$ in Pineau's analysis for an isolated spheroid. This suggests that the arguments of the paragraph which follows have a rather broad heuristic value.

In the mutual compensation of terms, only one term has survived. It is obtained from the term $-c_{11} \delta^{2} m \Sigma$ in equation (22), which appears in the energy density in a lateral $\gamma$ sheet. The physical origin of this term is clear. Suppose a small tensile stress $\sigma$ is applied. This stress will make a substantial first-order contribution to the energy density only in those regions which already have substantial strains in the longitudinal direction, i.e. in the lateral $\gamma$ sheets. This contribution to the energy density is $\frac{1}{2} c_{11} e_{\gamma \mathrm{L}}^{2}$, and the increase in this energy density is

$$
\Delta W_{0}=c_{11} \epsilon_{\mathrm{iL}}\left(\mathrm{d} e_{\mathrm{\gamma}} / \mathrm{d} \sigma\right) \sigma
$$

This is of the order of $\sigma \delta$. The only other term of this order is the contribution $-p_{\mathrm{n}}\left[e_{\mathrm{n}}\right]$ to the thermodynamic pressure on the lateral interfaces. The present calculation shows that if $\gamma^{\prime}$ and $\gamma$ have the same elastic constants, the contribution of $\sigma$ to $[W]$ on a lateral interface is exactly equal to the contribution of $\sigma$ to $-p_{\mathrm{n}}\left[\mathcal{c}_{\mathrm{n}}\right]$ on a transverse interface.

However, the thin lateral sheets of $\gamma$ are constrained by the cube of $\gamma^{\prime}$, and so, in general

$$
\begin{gathered}
\mathrm{d} e_{\gamma \mathrm{L}} / \mathrm{d} \sigma=1 /(1+m) c_{11} \\
\approx(1-m) / c_{11} .
\end{gathered}
$$

There is no corresponding term in $-p_{\mathrm{n}}\left[e_{\mathrm{n}}\right]$ on the transverse interfaces, and the uncompensated term $-m e_{\mathrm{L}} \sigma \approx-m \delta \sigma$ in equations (33) and (34) drives the rafting process. On rafting, the lateral interfaces sweep out a volume $v w^{v+1} a^{v+1} \epsilon$, and the energy release is $v w^{v+1} a^{v+1} \delta \epsilon m \sigma$, in agreement with equation (32).

We now enquire whether the presence of the pincushion stresses affects this argument. On the interfaces, the pincushion stresses of order $\tau c_{11}$ alter $\left[e_{\mathrm{n}}\right]$ by a quantity of the order of $m \tau$, which is negligible. So far as the term (39) effective on the lateral interfaces is concerned, the contribution is proportional to $e_{\gamma \mathrm{L}}$, the longitudinal strain present in a longitudinal sheet of $\gamma$ before the application of external stress. The pincushion stresses alter this strain by a fraction of the order of $\tau$. The uncompensated term $-m_{; \mathrm{L}} \sigma$ is therefore altered by a quality of the order of $\tau m e_{\gamma \mathrm{L}} \sigma$, which is negligible.

Since the contribution of fibre reinforcement to the stiffness of a composite depends on the angle between the fibre axes and the tensile axis, one might expect that the driving force for rafting under an applied stress $\sigma$ would contain a term in $\sigma^{2}$. No such term appears in our calculation. It is difficult to find a simple argument for or against its presence when the structure is one of cubes of $\gamma^{\prime}$ separated by thin sheets of $\gamma$. Pineau's calculations [10] for an isolated spheroid indicate, as would be expected, that platelets will be formed under high stresses, regardless of the signs of the misfit, of the difference of elastic constants, or of the stress, because the softer component is then free to draw energy from the loading system.

Since these effects are even in misfit and difference of elastic constants, and vanish when these quantities vanish, they will not appear in our linearized theory.

\section{ALLOWANCE MADE FOR POISSON CONTRACTION}

The regular cubical arrays of cubes before rafting and of rectangular parallelepipeds after rafting are stabilized by the high value of the anisotropy constant $2 c_{44} /\left(c_{11}-c_{12}\right)$. In the limit of a high volume fraction of $\gamma^{\prime}$, the elastic strains are composed of extensions and compressions along the cube axes. Shear strains which lead to energy densities proportional to $c_{44}$ are present only in negligibly small regions with volumes proportional to $\tau^{2}$. The rest of the material behaves like an isotropic medium with Young's modulus $\left(c_{11}^{2}+c_{11} c_{12}-2 c_{12}^{2}\right) /\left(c_{11}+c_{12}\right)$ or $\left(c_{11}^{\prime 2}+c_{11}^{\prime} c_{12}^{\prime}-2 c_{12}^{\prime 2}\right) /\left(c_{11}^{\prime}+c_{12}^{\prime}\right)$ and Poisson's ratio $c_{12} /\left(c_{11}+c_{12}\right)$ or $c_{12}^{\prime}\left(c_{11}^{\prime}+c_{12}^{\prime}\right)$.

We consider the three-dimensional case, and make allowance for the pincushion stresses and the Poisson contractions. Equations (7)-(10) remain valid. 
The stresses in the various regions may be written: in transverse $\gamma$ plates--

$$
\begin{aligned}
& \sigma_{\eta \mathrm{L}}=c_{11} e_{\gamma \mathrm{L}}+c_{12} e_{\gamma \mathrm{T}}+c_{12} e_{1 \mathrm{~T}} \\
& \sigma_{\eta \mathrm{T}}=c_{11} e_{\gamma \mathrm{T}}+c_{12} e_{\eta \mathrm{L}}+c_{12} e_{1 \mathrm{~T}} \\
& \sigma_{\mathrm{IT}}=c_{11} e_{1 \mathrm{~T}}+c_{12} e_{i \mathrm{~L}}+c_{12} e_{7 \mathrm{~T}}
\end{aligned}
$$

in $\gamma^{\prime}-$

$$
\begin{gathered}
\sigma_{\gamma^{\prime} \mathrm{L}}=c_{11}^{\prime} e_{\gamma^{\prime} \mathrm{L}}+2 c_{12}^{\prime} e_{\gamma^{\prime} \mathrm{T}} \\
\sigma_{\gamma^{\prime} \mathrm{T}}=c_{11}^{\prime} e_{\gamma^{\prime} \mathrm{T}}+c_{12}^{\prime} e_{\gamma^{\prime} \mathrm{L}}+c_{12}^{\prime} e_{\gamma^{\prime} \mathrm{T}}
\end{gathered}
$$

in normal $\gamma$ plates-

$$
\begin{gathered}
\sigma_{3 \mathrm{~L}}=c_{11} e_{3 \mathrm{~L}}+2 c_{12} e_{; \mathrm{T}} \\
\sigma_{3 \mathrm{~T}}=c_{11} e_{\gamma \mathrm{T}}+c_{12} e_{3 \mathrm{~T}}+c_{12} e_{3 \mathrm{~L}} .
\end{gathered}
$$

The conditions of equilibrium are

$$
\begin{gathered}
\sigma_{1 \mathrm{~T}}=0 \\
\sigma_{3 \mathrm{~L}}=\sigma \\
\tau(1+\delta) \sigma_{\gamma \mathrm{T}}+\tau(1+\delta) \sigma_{3 \mathrm{~T}}+(1+\delta)^{2} \sigma_{r \mathrm{~T}}=0 \\
2 \tau(1+\delta) \sigma_{\gamma \mathrm{L}}+(1+\delta)^{2} \sigma_{\gamma \mathrm{L}}=(1+\delta+\tau)^{2} \sigma .
\end{gathered}
$$

Again, $\delta$ may be neglected in the last two equations. We rewrite these, using equations (7)-(10) and the last remark, to obtain, with

$$
c_{11}^{\prime}=(1+m) c_{11}
$$

and

$$
c_{12}^{\prime}=(1+n) c_{12},
$$

results of equations (A7)-(A28).

The previous calculations may now be repeated, remembering that if the principal strains are $e_{1}, e_{2}$ and $e_{3}$ the energy density is now

$$
W=\frac{1}{2} c_{11}\left(e_{1}^{2}+e_{2}^{2}+e_{3}^{2}\right)+c_{12}\left(e_{2} e_{3}+e_{3} e_{1}+e_{1} e_{2}\right) .
$$

The final result is that the energy dissipated when a cube of $\gamma^{\prime}$ of dimensions $w a \times w a \times w a$ becomes a raft of dimensions

$$
\begin{aligned}
& (1+\epsilon) w a \times(1+\epsilon) w a \times(1-2 \epsilon) w a \text { is } \\
& -2 w^{3} a^{3} \epsilon \frac{\left(m c_{11}-n c_{12}\right)\left(c_{11}+2 c_{12}\right)}{c_{11}\left(c_{11}-c_{12}\right)} \delta \sigma .
\end{aligned}
$$

Here

$$
\begin{aligned}
& \delta=\frac{2\left(a^{\prime}-a\right)}{a^{\prime}+a} \\
& m=\frac{c_{11}^{\prime}-c_{11}}{c_{11}} \\
& n=\frac{c_{12}^{\prime}-c_{12}}{c_{12}} .
\end{aligned}
$$

We confirm that equation (32) with $v=2$ and equation (49) with $c_{12}=c_{12}^{\prime}=0$ agree.

Since elastic stability requires $c_{11}>0$, $c_{11}+2 c_{1},>0, c_{11}-c_{1}>0$ [22], the sign of equation
(49) is determined by that of $m c_{11}-n c_{12}$. It follows that the inclusion is harder or softer than the matrix if it has a larger or smaller value of the constant $c_{11}-c_{12}$. This is in agreement with the result of Johnson et al. [23] that for an isolated spheroid under uniaxial stress along a cube axis in a medium with cubic elastic anisotropy the precipitate shape is determined by $\Delta c_{11}-\Delta c_{12}$.

\section{THE INFLUENCE OF PLASTIC DEFORMATION IN THE MATRIX}

We have seen that, in the absence of plastic strain, the driving force for rafting has no term independent of $m$. Socrate and Parks [13] found that after appreciable plastic strain in the $\gamma$ matrix there was a term in $\sigma \delta$ which outweighed all others, and led to the formation of rafts when $\sigma \delta$ was negative.

We can understand this term in the following way. Socrate and Parks [24] not only allowed for the local increase of $p_{n}$ during plastic flow, but also included the plastic strain in the $\gamma$ sheet in $\left[e_{\mathrm{n}}\right]$. If one interprets equation (12) naively as meaning that when the interface sweeps out unit volume, stored elastic energy $[W]$ is released, while the normal traction $p_{\mathrm{n}}$ does work $p_{\mathrm{n}}\left[e_{\mathrm{n}}\right]$, then it might seem that prior plastic deformation should not contribute to $\left[e_{n}\right]$. Work hardening is not considered, and so prior plastic deformation on one side of an interface does not alter the state of the material in which it has occurred, and does not alter the changes of energy which occur when the interface moves. In the present case, however, the dislocations which caused the prior plastic strain have not moved out of the neighbourhood of the interface. They are stored in the interface. When the interface moves, these dislocations move, and the total volume which has been plastically strained is changed. The strain involved is not plastic, but anelastic, and, under the conditions assumed in the model, reversible.

The effect of this on the rafting behaviour follows immediately from the discussion below equation (32). The elastic constant mismatch between inclusion and matrix is no longer important. The mechanical properties of the plastic $\gamma$ phase no longer approximate closely to those of the $\gamma^{\prime}$ phase, which is still elastic.

Flow begins in those sheets in which the shear component of the applied stress adds to that produced by the coherency stresses. If $\sigma \delta$ is positive, these will be the lateral sheets, in the more usual case of negative $\sigma \delta$, these will be the transverse sheets. These plastic flows will upset the balance between the thermodynamic pressures tending to move the lateral and the transverse interfaces outwards. With positive $\sigma \delta$, plastic flow $\epsilon_{\mathrm{p}}$ in the lateral $\gamma$ sheets reduces the energy density in the lateral sheets by an amount of order $\epsilon_{\mathrm{p}} \sigma$, thereby reducing the outward thermodynamic pressure on the lateral interfaces, while the 
thermodynamic pressure on the transverse interfaces is unaffected by the plastic llow over the lateral interfaces. So, if $\sigma \delta$ is positive, needles are formed parallel to the axis of stress. If $\sigma \delta$ is negative, plastic flow begins outside the transverse interfaces. If these interfaces are displaced outwards, the volume of the plastic region is reduced, and the work done by the external forces is reduced by an amount $\epsilon_{\mathrm{p}} \sigma$ per unit volume swept out by these interfaces. The thermodynamic pressure on these interfaces is reduced, while that on the transverse interfaces is unaltered. Rafts are formed normal to the stress axis.

These rules agree with those following from the calculations of Socrate and Parks [13], and will apply when $\left|\epsilon_{\mathrm{p}} \sigma\right|>|m \delta \sigma|$, i.e.

$$
\left|\epsilon_{\mathrm{p}}\right|>|m \delta| \text {. }
$$

When the Pineau and the Socrate Parks rules disagree, which will be the case when $m$ is negative, the sense of rafting will reverse when the creep strain reaches a value of this order.

Such a reversal is actually observed in the computations of Socrate and Parks [13] in their Fig. 11, which applies to the (isotropic) case with $m=-0.089, \delta=+0.0002$. Their numerical treatment does not provide any obvious interpretation of such a reversal, and they attribute it to some unexplained failure of the numerical process arising from the very small value of $\delta$ : "the misfit chosen for this hypothetical alloy is exceedingly small-the misfit strains and the elastic strains have comparable magnitude. It is obvious that, in the limit of zero misfit, quantities normalized by the misfit itself lose their significance". In our treatment, a reversal occurring within the (very wide) range of plastic strains for which they made computations is explicitly predicted. The concordance of their numerical results and our analytical results and theoretical arguments should lend confidence to both.

\section{CONCLUSIONS}

(1) For the models consisting of square or cubical particles of $\gamma^{\prime}$ arranged in a simple square or cubical array and separated by thin layers of $\gamma$, there is no essential difference between the behaviours in two and three dimensions, or between the behaviours of a cubical particle in a cubic array and that of an isolated spherical particle.

(2) The driving force for rafting in the elastic regime is proportional to the product of the lattice misfit, the difference of elastic stiffnesses and the applied stress, leading to the formation of needles when all of these parameters are positive.

(3) The relevant elastic stiffness is the modulus $c_{11}-c_{12}$.

(4) In the elastic regime, there is no term in the driving force which is proportional to the square of the applied stress.
(5) If plastic deformation occurs in $\gamma$, this deformation should be regarded as anelastic and reversible.

(6) When the plastic strain exceeds $|m \delta|$, the driving force leads to the formation of platelets normal to the stress axis when the product of tensile stress and misfit is negative. If $m$ is negative, the direction of rafting reverses when the strain is of this order, as appears in the numerical analysis of Socrate and Parks.

Acknowledgement-We are grateful to a reviewer for suggestions as to the presentation of this paper.

\section{REFERENCES}

1. G. A. Webster and C. P. Sullivan, J. Inst. Metals 95, $138,(1967)$.

2. C. P. Sullivan, B. J. Piearcey and G. A. Webster, J. Inst. Metals 96, 274 (1968).

3. J. K. Tien and S. M. Copley, Metall. Trans. 2, 215 (1971).

4. J. K. Tien and S. M. Copley, Metall. Trans. 2, 543 (1971).

5. T. Miyazaki, K. Nakamura and H. Mori, J. Mater. Sci. 14, 1827 (1979).

6. T. M. Pollock and A. S. Argon, Acta metall. mater. 40, 1 (1992)

7. W. Schneider, J. Hammer and H. Mughrabi, in Superalloys 1992 (edited by S. D. Antolovich et al.), p. 589. The Minerals, Metals and Materials Society, Warrendale, PA (1992)

8. A. Fredholm and J. L. Strudel, in Superallovs 1984 (edited by M. Gell et al.), p. 211. Met. Soc. AIME, Warrendale, PA (1984)

9. A. Pineau, Acta metall. 24, 559 (1976).

10. J. D. Eshelby, Proc. R. Soc. A241, 376 (1952).

11. J. D. Eshelby, Prog. Solid Mech. 2, 87 (1961).

12. A. G. Khachaturyan. S. V. Semenovskaya and J. W. Morris, Acta metall. 36, 1563 (1988).

13. S. Socrate and D. M. Parks, Acta metall. mater. 41, 2185 (1993)

14. G. Colonnetti, Atti Accad. Naz. Lincei Rc. 24/1, 404 (1915).

15. F. R. N. Nabarro, Phil. Mag. Lett. in press.

16. A. G. Khachaturyan, Theory of Structural Transformations in Solids, p. 216. John Wiley Interscience, New York (1983)

17. J. N. Goodier, Phil. Mag. 23, 1017 (1937).

18. M. M. Crum. private communication, see, F. R. N. Nabarro, Proc. R. Soc. A125, 519 (1940).

19. K. Robinson, J. appl. Phys. 22, 1045 (1951).

20. J. D. Eshelby, in Inelastic Behaviour of Solids (edited by M. E. Kanninen et al.). McGraw Hill, New York (1969).

21. J. D. Eshelby, J. Elasticity 5, 321 (1975).

22. J. E. Nye, Physical Properties of Crystals, p. 142. Oxford, Clarendon Press (1960).

23. W. C. Johnson, M. B. Berkenpas and D. E. Laughlin, Acta metall. 36, 3149 (1988)

24. S. Socrate and D. M. Parks, private communication.

\section{APPENDIX}

When the Poisson contractions and the pincushion stresses are neglected, the squares of the strain components are given to the appropriate order by

$\left(e_{i 1} / \delta\right)^{2}=1-2 v \tau+2 \Sigma-2 m \Sigma-2 v \tau \Sigma+\Sigma^{2}-2 m \Sigma^{2}$

$$
\left(e_{\mathrm{r}} / \delta\right)^{2}=1-2 v \tau
$$




$$
\begin{gathered}
\left(e_{r \cdot \mathrm{L}} / \delta\right)^{2}=-2 v \tau \Sigma+\Sigma^{2}-2 m \Sigma^{2} \\
\left(e_{\gamma} \mathrm{\tau} / \delta\right)^{2}=0
\end{gathered}
$$

and

$$
\left(e_{\mathrm{TT}} / \delta\right)^{2}=0
$$

while

$$
\left(e_{3 \mathrm{~L}} / \delta\right)^{2}=\Sigma^{2} .
$$

In the general case, the stresses are given by

$$
\begin{array}{r}
\sigma_{\gamma_{\mathrm{L}}} / \delta=c_{11}(1+\zeta)+c_{12}(1+\xi)+c_{12}\left(e_{1 \mathrm{~T}} / \delta\right) \\
\sigma_{\gamma_{\mathrm{T}}} / \delta=c_{11}(1+\xi)+c_{12}(1+\zeta)+c_{12}\left(e_{1 \mathrm{~T}} / \delta\right) \\
\sigma_{1 \mathrm{~T}} / \delta=c_{11}\left(e_{1 \mathrm{~T}} / \delta\right)+c_{12}(1+\zeta)+c_{12}(1+\xi) \\
\sigma_{\gamma^{\mathrm{L}} \mathrm{L}} / \delta=(1+m) c_{11} \zeta+2(1+n) c_{12} \xi \\
\sigma_{\gamma_{\mathrm{T}} \mathrm{T}} / \delta=(1+m) c_{11} \xi+(1+n) c_{12} \zeta+(1+n) c_{12} \xi \\
\sigma_{3 \mathrm{~L}} / \delta=c_{11}\left(e_{3 \mathrm{~L}} / \delta\right)+2 c_{12}(1+\xi) \\
\sigma_{3 \mathrm{~T}} / \delta=c_{11}(1+\xi)+c_{12}(1+\xi)+c_{12}\left(e_{3 \mathrm{~L}} / \delta\right)
\end{array}
$$

together with

$$
\begin{gathered}
\sigma_{1 \mathrm{~T}}=0 \\
\sigma_{3 \mathrm{~L}}=0 \\
\tau \sigma_{\mathrm{iT}}+\tau \sigma_{3 \mathrm{~T}}+\sigma_{i \mathrm{~T}}=0
\end{gathered}
$$

and

$$
2 \tau \sigma_{\gamma \mathrm{L}}+\sigma_{i \mathrm{~L}}=(1+2 \tau) \sigma .
$$

Substituting the first seven into the last four

$$
\begin{gathered}
c_{11}\left(e_{1 \mathrm{~T}} / \delta\right)+c_{12}(1+\zeta)+c_{12}(1+\xi)=0 \\
c_{11}\left(e_{3 \mathrm{~L}} / \delta\right)+2 c_{12}(1+\xi)=\sigma \\
c_{11} \tau(1+\xi)+c_{12} \tau(1+\zeta)+c_{12} \tau\left(e_{1 \mathrm{~T}} / \delta\right) \\
+c_{11} \tau(1+\xi)+c_{12} \tau(1+\xi)+c_{12} \tau\left(e_{3 \mathrm{~L}} / \delta\right) \\
+(1+m) c_{11} \xi+(1+n) c_{12} \zeta+(1+n) c_{12} \xi=0
\end{gathered}
$$

and

$$
2 c_{11} \tau(1+\zeta)+2 c_{12} \tau(1+\xi)+2 c_{12} \tau\left(e_{1 T} / \delta\right)
$$$$
+(1+m) c_{11} \zeta+2(1+n) c_{12} \xi=(1+2 \tau) \sigma .
$$

From equation (A16)

$$
\left(e_{\mathbf{I T}} / \delta\right)=-\frac{c_{12}}{c_{1 !}}(2+\xi+\zeta) .
$$

From equation (A19)

$$
E_{3 L} / \delta=\left(\sigma / c_{11}\right)-\frac{2 c_{12}}{c_{11}}(1+\xi)
$$

Substituting into (A18) leads to

$$
\begin{aligned}
& c_{11} \tau(1+\xi)+c_{12} \tau(1+\zeta)-\frac{c_{12}^{2} \tau}{c_{11}}(2+\xi+\zeta) \\
& \quad+c_{11} \tau(1+\xi)+c_{12} \tau(1+\xi)+\frac{c_{12} \tau}{c_{11}} \sigma-\frac{2 c_{12}^{2} \tau}{c_{11}}(1+\xi)
\end{aligned}
$$$$
+(1+m) c_{11} \xi+(1+n) c_{12} \zeta+(1+n) c_{12} \xi=0 .
$$

Substituting into (A21) leads to

$$
\begin{aligned}
2 c_{11} \tau(1 & +\zeta)+2 c_{12} \tau(1+\xi)-\frac{c_{12}^{2} \tau}{c_{11}}(2+\xi+\zeta) \\
& +(1+m) c_{11} \zeta+2(1+n) c_{12} \xi=(1+2 \tau) \sigma .
\end{aligned}
$$

An elementary but tedious calculation leads to

$$
\begin{aligned}
& -\frac{2\left(c_{11}-c_{12}\right)}{c_{11}} \tau-\frac{c_{12} \sigma}{R}+\frac{m c_{12} \sigma}{R}-\frac{(n-m)\left(c_{11}^{2} c_{12}+\right.}{R^{2}} \\
& \frac{\left.-c_{12}\right)}{c_{11}} \tau+\frac{\left(c_{11}+c_{12}\right) \sigma}{R}-\frac{m\left(c_{11}+c_{12}\right) \sigma}{R}+\frac{(n-m)(4)}{}
\end{aligned}
$$

with

$$
R=c_{11}^{2}+c_{11} c_{12}-2 c_{12}^{2}
$$

